\title{
Circulating IL-37 levels are elevated in patients with hypertension
}

\author{
JING YE ${ }^{1,2 *}$, YUAN WANG $^{3 *}$, ZHEN WANG $^{2}$, YINGZHONG LIN ${ }^{1}$, LING LIU $^{1}$, QI ZHOU ${ }^{4}$ \\ MENGLONG WANG $^{2}$, YAO XU ${ }^{2}$, DI YE ${ }^{2}$, JISHOU ZHANG ${ }^{2}$, JUN WAN ${ }^{2}$ and QINGWEI JI ${ }^{1}$ \\ ${ }^{1}$ Department of Cardiology, People's Hospital of Guangxi Zhuang Autonomous Region, Nanning, Guangxi 530021; \\ ${ }^{2}$ Department of Cardiology, Renmin Hospital of Wuhan University, Hubei Key Laboratory of Cardiology, \\ Cardiovascular Research Institute, Wuhan University; ${ }^{3}$ Department of Thyroid Breast Surgery, \\ Renmin Hospital of Wuhan University, Wuhan, Hubei 430060; ${ }^{4}$ Department of Cardiology, \\ Beijing Anzhen Hospital, Capital Medical University, Beijing Institute of Heart, Lung and Blood Vessel Diseases, \\ Key Laboratory of Remodeling-Related Cardiovascular Disease, Ministry of Education, Beijing 100029, P.R. China
}

Received November 26, 2019; Accepted July 23, 2020

DOI: $10.3892 / \mathrm{etm} .2021 .9990$

\begin{abstract}
Interleukin-37 (IL-37) has been reported to be closely linked to vascular diseases, including atherosclerosis and aortic calcification. The present study aimed to assess the expression levels of IL-37 in patients with hypertension. Blood samples were collected from control subjects $(n=20)$ and patients with hypertension $(\mathrm{n}=45)$. Subsequently, macrophages, lymphocytes and dendritic cells were individually isolated and the mRNA expression of IL-37 was measured. In addition, the circulating IL-37 levels in control subjects $(n=30)$ and patients with hypertension $(n=334)$ were assessed. Furthermore, all patients who were subjected to detection of circulating IL-37 underwent ambulatory blood pressure monitoring. The results suggested that the mRNA levels of IL-37 in macrophages, but not in lymphocytes and dendritic cells, isolated from patients with hypertension were markedly elevated compared with those in cells isolated from control subjects. Circulating IL-37 levels were increased in patients with hypertension compared with those in control subjects and positively correlated with systolic and diastolic blood pressure in patients with hypertension. No differences were observed between patients with dipper hypertension and patients with
\end{abstract}

Correspondence to: Professor Qingwei Ji, Department of Cardiology, People's Hospital of Guangxi Zhuang Autonomous Region, 6 Taoyuan Road, Nanning, Guangxi 530021, P.R. China E-mail: jqw124@163.com

Professor Jun Wan, Department of Cardiology, Renmin Hospital of Wuhan University, Hubei Key Laboratory of Cardiology, Cardiovascular Research Institute, Wuhan University, 9 Zhangzhidong Road, Wuhan, Hubei 430060, P.R. China

E-mail:wanjun@whu.edu.cn

${ }^{*}$ Contributed equally

Key words: hypertension, interleukin-37, macrophages, dipper hypertension, non-dipper hypertension non-dipper hypertension. In addition, patients with hypertension with a smoking habit, type 2 diabetes mellitus and carotid atherosclerotic plaque (CAP) exhibited higher IL-37 levels. IL-37 levels were positively correlated with creatinine, C-reactive protein and homocysteine levels. Furthermore, the results of a linear regression analysis suggested that IL-37 levels were independently associated with the presence of CAP. In conclusion, IL-37 levels are increased in patients with hypertension and may be associated with the onset of CAP.

\section{Introduction}

Hypertension is a complex clinical syndrome that is usually characterized by abnormally elevated systolic blood pressure (SBP) and/or diastolic blood pressure (DBP) and comprises a wide range of patients and various clinical complications. Various pathological mechanisms have been indicated to be closely linked to the occurrence and progression of hypertension and target organ injury mediated by hypertension, among which inflammation is one of the most important mechanisms (1-3).

Interleukin-37 (IL-37) belongs to the IL-1 family and is the only anti-inflammatory factor in this family (4). IL-37 is expressed in tissues and organs of a variety of mammals but is not observed in mice; in addition, IL-37 is mainly secreted by immune cells such as macrophages and dendritic cells and may also be secreted in small amounts by endothelial cells and smooth muscle cells $(4,5)$. IL-37 is able to regulate the differentiation of various immune cells, affect the expression of downstream inflammatory factors and participate in the development and progression of a variety of diseases by binding with IL-18 receptor (IL-18R) $\alpha$ and IL-1R8 receptors and further activating the Toll-like receptor 4 pathway (6-8).

IL-37 has been the focus of recent research and increasing evidence has confirmed that it is closely associated with the progression of cardiovascular disease $(9,10)$. IL-37 has been indicated to alleviate the progression of atherosclerosis and enhance the stability of plaques in apolipoprotein E knockout mice fed a high-fat diet by inhibiting T-lymphocyte differentiation, dendritic cell maturation and vascular smooth muscle 
cell apoptosis (11-13). In another study, IL-37 was reported to have no effect on atherosclerosis development in low-density lipoprotein receptor-deficient mice receiving a high-fat diet, although the local inflammation in the blood vessels was reduced (14). In addition, IL-37 may reduce the area of myocardial infarction and reverse cardiac remodeling by inhibiting the maturation of dendritic cells (15). Furthermore, treatment with exogenous IL-37 alleviates myocardial injury induced by ischemia/reperfusion via the upregulation of IL-10 expression (16). However, the association between IL-37 and hypertension has remained elusive and the purpose of the present study was to observe IL-37 expression in the context of hypertension.

\section{Materials and methods}

Blood sample collection. As described in a previous study, patients with secondary hypertension, coronary artery disease, heart failure, stroke, valvular heart disease, collagen disease, advanced liver disease, renal failure, malignant disease, septicemia, or other inflammatory diseases were excluded from the present study (17). The study was approved by the ethics committee of the People's Hospital of Guangxi Zhuang Autonomous Region (Nanning, China; approval no. 2015-16 of the National Natural Science Foundation of China), and written informed consent was obtained from the participants.

The diagnosis of hypertension was based on the results of ambulatory blood pressure monitoring (ABPM). In brief, blood pressure values for 48 time-points were collected over a 24-h period and patients with abnormally high blood pressure (SBP/DBP $\geq 140 / 90 \mathrm{mmHg}$ from 7 am to $0 \mathrm{am}$ and SBP/DBP $\geq 125 / 85 \mathrm{mmHg}$ from 0 am to $7 \mathrm{am}$ ) detected at $>25 \%$ of the time-points were diagnosed with hypertension $(18,19)$. Secondary hypertension, hyperthyroidism, white-coat syndrome, elevated blood pressure due to emotional agitation, and all other diseases and conditions that may affect blood pressure, were excluded (19).

Within 2 months from October 2018 to January 2020, a total of 110 subjects who were admitted for physical examination at the medical center were enrolled. The subjects were divided in to a non-hypertension group (control subjects, $n=40$ ) and a hypertension group $(n=70)$ based on the results of ABPM. The monocytes and T-lymphocytes were separated from the fresh blood samples and then stimulated to differentiate into macrophages, dendritic cells and mature T-lymphocytes. In brief, peripheral blood mononuclear cells were isolated from each blood sample after the red blood cells were lysed. Subsequently, the T-lymphocytes and monocytes were positively selected using human CD4 (cat. no. 130-045-101) and CD11b (cat. no. 130-049-601) magnetic beads and an autoMACS separator (cat. no. 130-049-601; all from Miltenyi Biotech) (20). The monocytes were treated with human macrophage colony-stimulating factor (M-CSF; $50 \mathrm{ng} / \mathrm{ml}$; cat. no. AF-300-25; PeproTech) or human granulocyte-M-CSF (50 ng/ml; cat. no. 900-K30; PeproTech) for 9 days to stimulate their differentiation into macrophages and dendritic cells, respectively. The T lymphocytes were incubated with Cell Stimulation Cocktail ( $\mu \mathrm{l} / \mathrm{ml}$; cat. no. 00-4975-93; eBioscience), which was composed of $40.5 \mu \mathrm{mol} / 1$ phorbol myristate acetate, $670 \mu \mathrm{mol} / 1$ ionomycin, $5.5 \mu \mathrm{mol} / 1$ brefeldin and
$1 \mu \mathrm{mol} / 1 \mathrm{monensin}$ for $4 \mathrm{~h}$ to promote maturation. IL-37 mRNA expression in each isolate of immune cells from all subjects was detected.

From March 2017 to January 2020, 491 hospitalized subjects were enrolled in the present study. According to the above-mentioned criteria, 67 subjects were excluded from the study. Blood samples were collected from the remaining 424 subjects (including 90 subjects without hypertension and 334 patients with hypertension) in a fasted state in the morning following admission by experienced nurses for the collection of plasma samples and measurement of IL-37 levels.

Reverse transcription-quantitative (RT-q)PCR detection of IL-37 mRNA expression. The macrophages, T-lymphocytes and dendritic cells were lysed using TRIzol reagent (cat no. T9424; Sigma-Aldrich; Merck KGaA) according to the manufacturer's protocol, and the total mRNA of each sample was individually extracted. Subsequently, the complementary (c)DNA was obtained by RT using $2 \mu \mathrm{g}$ of total mRNA and a Transcriptor First Strand cDNA Synthesis kit (cat. no. 04896866001; Roche Molecular Systems, Inc.) at $92^{\circ} \mathrm{C}$ for $5 \mathrm{~min}$. The IL-37 mRNA levels were detected by PCR amplification using SYBR Green (cat no. 04707516001; Roche Molecular Systems, Inc.) and were normalized to GAPDH levels using the $2^{-\Delta \Delta C q}$ method (21). The following thermocycling conditions were used for the PCR: 35 cycles at $92^{\circ} \mathrm{C}$ for $30 \mathrm{sec}, 58^{\circ} \mathrm{C}$ for $40 \mathrm{sec}$ and $72^{\circ} \mathrm{C}$ for $35 \mathrm{sec}$. The primers (Qingke Technology) used in the present study were as follows: IL-37 forward, 5'-AGTGCTGCTTAG AAGACCCGG-3' and reverse, 5'-AGAGTCCAGGACCAG TACTTTGTGA-3'; GAPDH forward, 5'-GAGTCAACGGAT TTGGTCGT-3' and reverse, 5'-GACAAGCTTCCCGTTCTC AG-3'.

Measurement of plasma IL-37 levels. The samples were obtained in sodium heparin vacutainers and centrifuged at $5,000 \mathrm{xg}$ for $15 \mathrm{~min}$. Plasma was obtained from each sample after centrifugation and was stored at $-80^{\circ} \mathrm{C}$ until further detection. Prior to investigation, the frozen plasma samples were thawed at $4^{\circ} \mathrm{C}$ and an ELISA (cat. no. 88-52103-22; Invitrogen; Thermo Fisher Scientific, Inc.) was performed to determine the IL-37 levels in each sample. All assays were performed in duplicate.

Echocardiography and $A B P M$. All of the control subjects and patients with hypertension received B-mode ultrasound examinations and underwent ABPM to identify whether the patients suffered from carotid atherosclerosis and/or non-dipper hypertension. The structural and functional changes in the heart may affect interleukin secretion. Therefore, all subjects underwent echocardiography, and subjects with significant cardiac structural and functional changes were excluded. Both of these tests were completed and analyzed by doctors with $>10$ years of experience. In addition, the 24-h coefficient of variation of SBP (CV-SBP), CV-DBP, night drop rate of SBP (NDR-SBP), NDR-DBP, average SBP in $24 \mathrm{~h}$ (A24h-MSBP), $24 \mathrm{~h}-\mathrm{MDBP}$, average active period of SBP (AP-SBP), AP-DBP, average passive period of SBP (PP-SBP) and PP-DBP were collected after the ABPM of each patient was completed.

Collection of patient information. Information on age, sex, smoking, drinking, body mass index (BMI) and medical 
Table I. Clinical characteristics of the control and hypertension groups.

\begin{tabular}{|c|c|c|c|c|}
\hline Characteristics & Normal range & Control $(n=40)$ & Hypertension $(n=70)$ & P-value \\
\hline Age (years) & - & $53.2 \pm 10.2$ & $56.2 \pm 11.5$ & 0.1735 \\
\hline Male sex & - & $25(62.5)$ & $40(50.0)$ & 0.6877 \\
\hline Smoking & - & $13(32.5)$ & $45(64.3)$ & 0.0016 \\
\hline Drinking & - & $9(22.5)$ & $20(28.6)$ & 0.6533 \\
\hline Obesity & - & $14(35.0)$ & $30(42.9)$ & 0.5433 \\
\hline $\mathrm{T} 2 \mathrm{DM}$ & - & $3(7.5)$ & $4(5.7)$ & 0.7033 \\
\hline HLP & - & $10(25.0)$ & $20(28.6)$ & 0.8246 \\
\hline CAP & - & $2(5.0)$ & $13(18.6)$ & 0.0800 \\
\hline HR (bpm) & $60-100$ & $72.5 \pm 10.6$ & $75.3 \pm 12.1$ & 0.2252 \\
\hline SBP (mmHg) & $90-140$ & $119 \pm 13$ & $155 \pm 26$ & $<0.0001$ \\
\hline $\mathrm{DBP}(\mathrm{mmHg})$ & $60-90$ & $76 \pm 12$ & $95 \pm 19$ & $<0.0001$ \\
\hline BMI $\left(\mathrm{kg} / \mathrm{m}^{2}\right)$ & $20-25$ & $23.2 \pm 3.5$ & $23.6 \pm 3.6$ & 0.5724 \\
\hline Glu (mmol/l) & $3.5-6.1$ & $4.6 \pm 0.6$ & $4.7 \pm 0.6$ & 0.4023 \\
\hline HbAlc $(\%)$ & 4-6 & $4.8 \pm 1.0$ & $4.9 \pm 1.1$ & 0.6366 \\
\hline $\mathrm{TC}(\mathrm{mmol} / \mathrm{l})$ & $3-5.2$ & $4.5 \pm 0.8$ & $4.7 \pm 1.0$ & 0.2817 \\
\hline TG (mmol/l) & $0.5-1.7$ & $1.1 \pm 0.5$ & $1.1 \pm 0.8$ & 0.8724 \\
\hline HDL-C (mmol/l) & $0.9-2.0$ & $1.1 \pm 0.4$ & $1.3 \pm 0.7$ & 0.3523 \\
\hline LDL-C (mmol/l) & $1.8-3.1$ & $2.2 \pm 0.6$ & $2.5 \pm 0.9$ & 0.5262 \\
\hline CREA $(\mu \mathrm{mol} / \mathrm{l})$ & $57-97$ & $66 \pm 14$ & $69 \pm 19$ & 0.2152 \\
\hline CRP (mg/l) & $<5$ & $0.6 \pm 0.8$ & $6.4 \pm 4.7$ & $<0.0001$ \\
\hline Hcy $(\mu \mathrm{mol} / \mathrm{l})$ & $0-15$ & $10.3 \pm 6.4$ & $11.1 \pm 7.1$ & 0.1952 \\
\hline \multicolumn{5}{|l|}{ Medical treatments } \\
\hline ACEI/ARB & - & $0(0.0)$ & $39(55.7)$ & $<0.0001$ \\
\hline$\beta$-blocker & - & $0(0.0)$ & $23(32.9)$ & $<0.0001$ \\
\hline $\mathrm{CCB}$ & - & $0(0.0)$ & $51(72.9)$ & $<0.0001$ \\
\hline Diuretics & - & $0(0.0)$ & $30(42.8)$ & $<0.0001$ \\
\hline$\alpha$-blocker & - & $0(0.0)$ & $6(8.6)$ & 0.0845 \\
\hline Statin & - & $4(10.0)$ & $36(51.4)$ & $<0.0001$ \\
\hline
\end{tabular}

Values are expressed as $\mathrm{n}(\%)$ or the mean \pm standard deviation. T2DM, type 2 diabetes mellitus; HLP, hyperlipidemia; CAP, carotid atherosclerotic plaque; HR, heart rate; SBP, systolic blood pressure; DBP, diastolic blood pressure; BMI, body mass index; Glu, fasting glucose; HbAlc, hemoglobin a1c; TC, total cholesterol; TG, total triglycerides; HDL-C, high-density lipoprotein cholesterol; LDL-C, low-density lipoprotein cholesterol; CREA, creatinine; CRP, C-reactive protein; Hcy, homocysteine; ACEI, angiotensin-converting enzyme inhibitor; ARB, angiotensin receptor blocker; $\mathrm{CCB}$, calcium channel blocker.

treatments was obtained when the patients' medical history was taken after admission. Information on SBP, DBP and heart rate (HR) was collected by the attending physician. The fasting glucose (Glu), hemoglobin A1c (HbAlc), total cholesterol (TC), total triglycerides (TG), high-density lipoprotein cholesterol (HDL-C), low-density lipoprotein cholesterol (LDL-C), creatinine (CREA), C-reactive protein (CRP), homocysteine (Hcy), sleep apnea hypopnea syndrome (SAHS) and hypoxemia-associated data were obtained after laboratory tests.

Statistical analysis. All of the data in the present study are expressed as the mean \pm standard deviation and were further analyzed using SPSS 26.0 statistical software (IBM Corp.). Discrete variables are expressed as n (\%) and were compared with Chi-square tests. For the continuous variables, Student's t-test was performed to compare the differences between
2 groups, and one-way analysis of variance followed by the Tukey's post hoc test was used for comparisons involving 3 groups. In addition, the correlations between IL-37 levels and SBP, DBP and clinical characteristics in patients with hypertension were calculated using Pearson's correlation. Furthermore, the influence of hypertension on IL-37 secretion and IL-37 levels on the presence of carotid atherosclerotic plaque (CAP) were identified by univariate analysis and subsequent multivariate linear regression analysis. $\mathrm{P}<0.05$ was considered to indicate statistical significance in all analyses.

\section{Results}

Clinical characteristics. The clinical characteristics of each group are listed in Table I. Compared with the control group, the hypertension group exhibited a higher rate of smoking, 
A

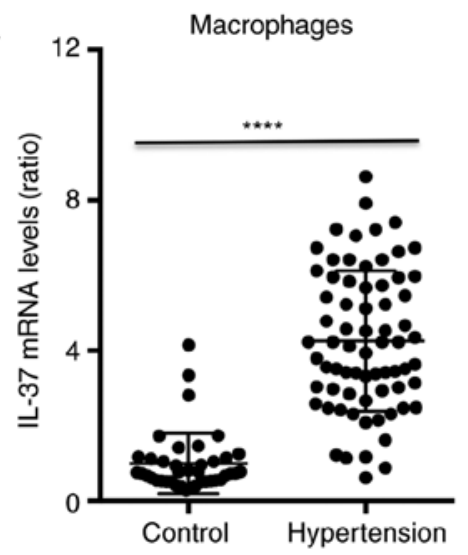

B

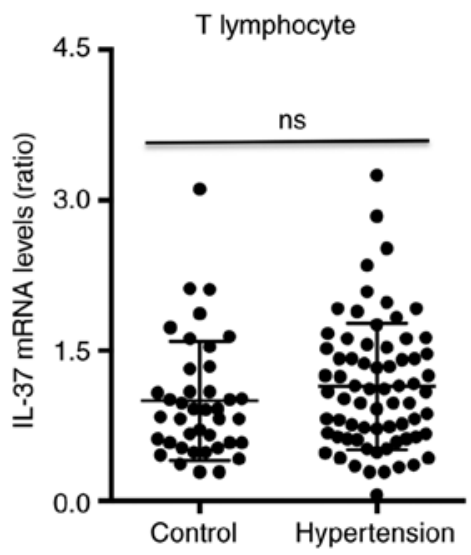

C

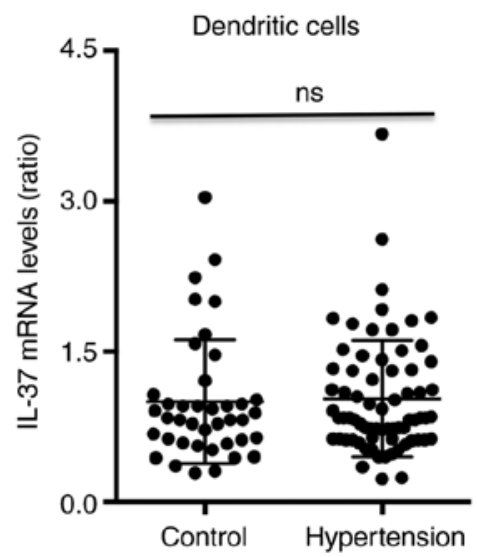

Figure 1. IL-37 mRNA expression in patients with hypertension. IL-37 mRNA expression was detected in (A) macrophages, (B) T lymphocytes and (C) dendritic cells from control subjects and patients with hypertension by reverse transcription-quantitative PCR. The results are presented as a ratio to the expression of the control group. ${ }^{* * * *} \mathrm{P}<0.0001$ vs. the control group. ns, no significance; IL, interleukin.

higher SBP and DBP and elevated levels of CRP. No significant inter-group differences were observed in other clinical characteristics, including age, sex, drinking, HR, presence of CAP, BMI, Glu, HbAlc, blood lipids, CREA and Hcy.

IL-37 mRNA levels in patients with hypertension and controls. The RT-qPCR results indicated that the average IL-37 mRNA expression in macrophages from hypertensive patients was increased $\sim 3.5$-fold compared with that in macrophages from control subjects (Fig. 1A). The IL-37 mRNA levels in T-lymphocytes and dendritic cells exhibited no differences between control subjects and patients with hypertension (Fig. 1B and C, respectively). In addition, a univariate analysis was performed to identify any factors affecting IL-37 expression in the entire cohort. The $\beta$-values, 95\% CI of $\beta$ and P-values are listed in Table II, and factors including macrophages, T-lymphocytes, dendritic cells, hypertension, smoking, drinking, obesity, CREA, CRP and Hcy in Table II were adjusted. The results suggested that macrophages, hypertension, smoking and CRP significantly affected IL-37 expression. These variables were used to further perform multivariate regression analysis and the results indicated that hypertension and macrophages were closely and independently associated with the expression of IL-37.

Clinical characteristics of subgroups of hypertension and controls. The clinical characteristics of each group are listed in Table SI. Compared with those in the control group, the hypertension group exhibited significantly higher rates of obesity, type 2 diabetes mellitus (T2DM), hyperlipidemia (HLP), SAHS and CAP, higher levels of SBP, DBP, BMI, Glu, HbAlc, TG, CREA, CRP and Hcy, and lower levels of HDL-C. A higher tendency of male sex, smoking and drinking was observed in the hypertension group, but it was not statistically significant compared with the control group. No differences in other clinical factors were observed between the two groups. In addition, the non-dipper group exhibited increased T2DM and CAP rates compared with those in the dipper group, and the dipper group exhibited a lower rate of CAP compared with the total group. No differences in terms of any other clinical characteristics, including age, sex, smoking, drinking,
HR, TC or HDL-C, were obtained among the control, dipper hypertension, non-dipper hypertension and total hypertension group.

Information on ABPM. The ABPM data for each group are listed in Table SII. The hypertension group had a higher NDR-SBP, NDR-DBP, A24h-SBP, A24h-DBP, AP-SBP, AP-DBP, PP-SBP and PP-DBP than the control group. Compared with the dipper group, the non-dipper group exhibited increases in the PP-SBP and PP-DBP and decreases in the NDR-SBP and NDR-SBP. No differences in A24h-SBP, A24h-DBP, AP-SBP and AP-DBP were observed between the dipper group and the non-dipper group, and no differences in CV-SBP and CV-DBP were observed among the four groups.

Plasma IL-37 concentration in patients with hypertension. Regarding the ELISA results, the IL-37 levels gradually increased in patients with grade I, II and III hypertension and those in all groups were higher than those in the control group (Fig. 2). In addition, no differences in plasma IL-37 levels were observed among the hypertension group, the dipper group and the non-dipper group, while the levels in all of these groups were significantly elevated compared with the levels in the control group (Fig. 3A). Furthermore, IL-37 levels were positively correlated with SBP and with DBP in patients with hypertension (Fig. 3B and C, respectively). A univariate analysis of variables affecting the plasma levels of IL-37 was then performed. The $\beta$ values, 95\% CI of $\beta$, and P-values are listed in Table III. The results suggested that CAP, hypertension, non-dipper hypertension, smoking, T2DM, HLP, CREA, CRP and Hcy had a significant influence on the plasma levels of IL-37. These variables were subjected to further multivariate regression analysis and the results suggested that CAP, hypertension, smoking, T2DM, HLP, CREA, CRP and Hcy were closely independently associated with the secretion of IL-37 into the plasma.

Smoking, T2DM and CAP influence circulating IL-37 levels in patients with hypertension. In addition, all patients with hypertension were divided into two groups according to sex, 
Table II. Logistic regression analysis of the influence of clinical factors on interleukin-37 mRNA expression in the cohort.

\begin{tabular}{|c|c|c|c|c|c|c|c|}
\hline \multirow[b]{2}{*}{ Factor } & \multirow[b]{2}{*}{ Cut-off value } & \multicolumn{3}{|c|}{ Univariate } & \multicolumn{3}{|c|}{ Multivariate } \\
\hline & & Adjusted $\beta$ & $95 \% \mathrm{CI}$ of $\beta$ & P-value & Adjusted $\beta$ & $95 \% \mathrm{CI}$ of $\beta$ & P-value \\
\hline Macrophages & Yes & 0.491 & $0.352-0.630$ & $<0.001$ & 0.305 & $0.226-0.484$ & $<0.001$ \\
\hline T-lymphocytes & Yes & 0.094 & $0.041-0.147$ & 0.098 & & & \\
\hline Dendritic cells & Yes & 0.033 & $0.010-0.056$ & 0.274 & & & \\
\hline Hypertension & Yes & 0.392 & $0.267-0.517$ & $<0.001$ & 0.196 & $0.101-0.291$ & 0.009 \\
\hline Smoking & Yes & 0.202 & $0.150-0.254$ & 0.029 & 0.101 & $0.051-0.151$ & 0.091 \\
\hline Drinking & Yes & 0.088 & $0.037-0.139$ & 0.339 & & & \\
\hline Obesity & Yes & 0.042 & $0.019-0.065$ & 0.618 & & & \\
\hline CREA & $>79 \mu \mathrm{mol} / \mathrm{l}$ & 0.007 & $-0.012-0.026$ & 0.512 & & & \\
\hline CRP & $>8.6 \mathrm{mg} / \mathrm{l}$ & 0.164 & $0.084-0.244$ & 0.004 & 0.078 & $0.037-0.119$ & 0.019 \\
\hline Hcy & $>13.5 \mu \mathrm{mol} / 1$ & -0.04 & $-0.023-0.015$ & 0.887 & & & \\
\hline
\end{tabular}

CREA, creatinine; CRP, C-reactive protein; Hcy, homocysteine.

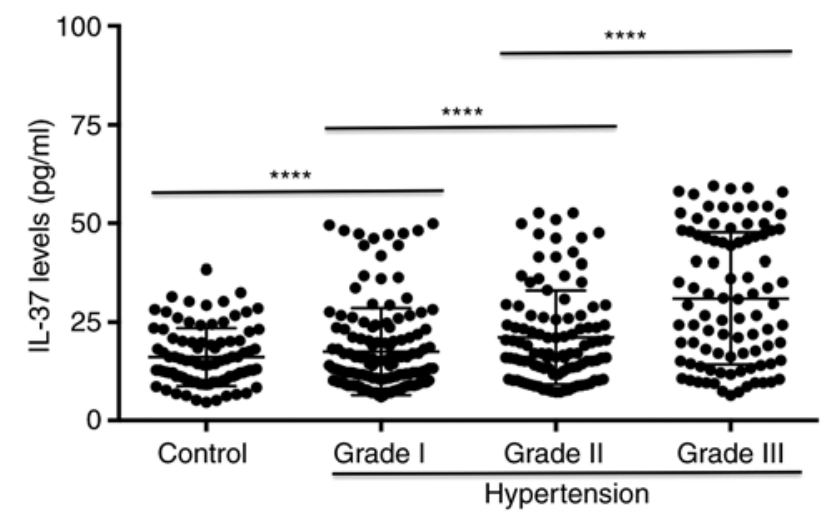

Figure 2. IL-37 levels in patients with hypertension. The plasma IL-37 levels in the control group, grade I group, grade II group and grade III group were detected. ${ }^{* * * *} \mathrm{P}<0.0001$ vs. the previous group. IL, interleukin.

smoking, drinking, obesity, T2DM, HLP, SAHS, hypoxemia and CAP and the serum levels of IL-37 were compared between them (Fig. 4). The results suggested that patients who smoked, those who had T2DM and those with CAP exhibited higher plasma IL-37 levels than patients who did not smoke or have T2DM or CAP (Fig. 4B, E and I). Other factors, including sex, drinking, obesity, hyperlipidemia, SAHS and hypoxemia, had no effects on the plasma levels of IL-37 in patients with hypertension (Fig. 4A, C, D, F and H).

Effects of IL-37 on the presence of CAP. The correlation between IL-37 levels and clinical characteristics was then analyzed. The results suggested that CREA, Hcy and CRP were all positively correlated with plasma IL-37 levels in individuals with hypertension (Fig. 5A-C). No significant correlations between plasma IL-37 levels and BMI, Glu, TC, LDL-C or HDL-C were observed (data not shown). Subsequently, a univariate linear regression analysis regarding the influence on the presence of CAP was performed for the variables which exhibited differences between the control group and the hypertension group or were demonstrated to be associated with the presence of CAP, including IL-37, hypertension, non-dipper status, age, male sex, smoking, drinking, obesity, T2DM, HLP, SAHS, hypoxemia, CREA, CRP and Hcy. The $\beta$-values, 95\% CI of $\beta$ and P-values are listed in Table IV. The results suggested that IL-37, hypertension, non-dipper status, age, smoking, T2DM, HLP, SAHS, hypoxemia and CRP had an influence on the occurrence of CAP. These variables provided in Table IV, including IL-37, hypertension, non-dipper status, age, sex, smoking, drinking, obesity, T2DM, HLP, SAHS, hypoxemia, CREA, CRP and Hcy, were all adjusted and used to further perform multivariate regression analysis and the results suggested that the presence of CAP was independently associated with IL-37, hypertension, non-dipper state, age, sex and smoking.

\section{Discussion}

The present study was the first to report that IL-37 mRNA expression was significantly increased in macrophages in patients with hypertension, and that circulating IL-37 levels were significantly increased in patients with hypertension and were positively correlated with SBP and DBP. Although non-dipper hypertension did not affect plasma IL-37 levels, smoking, T2DM and CAP were able to promote IL-37 expression. IL-37 levels were also positively correlated with CREA, CRP and Hcy levels in patients with hypertension, and elevated IL-37 levels were independently associated with the presence of CAP.

Numerous clinical studies have confirmed that IL-37 expression is elevated in a variety of cardiovascular diseases. In an early study, IL-37 levels were observed to be increased in the plasma and arteries of patients with arterial calcification (22). Subsequently, plasma IL-37 levels were indicated to be increased in patients with aortic calcification and aortic valve calcification $(23,24)$. In addition, IL-37 levels were elevated in patients with chronic heart failure and higher IL-37 levels suggested poor prognosis (25). Furthermore, substantial evidence has indicated that the plasma concentration of IL-37 was significantly increased in patients 
Table III. Logistic regression analysis of the effects of clinical factors on plasma interleukin-37 levels.

\begin{tabular}{|c|c|c|c|c|c|c|c|}
\hline \multirow[b]{2}{*}{ Factor } & \multirow[b]{2}{*}{ Cut-off value } & \multicolumn{3}{|c|}{ Univariate } & \multicolumn{3}{|c|}{ Multivariate } \\
\hline & & Adjusted $\beta$ & $95 \%$ CI of $\beta$ & P-value & Adjusted $\beta$ & $95 \% \mathrm{CI}$ of $\beta$ & P-value \\
\hline CAP & Yes & 0.377 & $0.219-0.535$ & $<0.001$ & 0.195 & $0.125-0.265$ & $<0.001$ \\
\hline Hypertension & Yes & 0.272 & $0.156-0.388$ & $<0.001$ & 0.152 & $0.106-0.197$ & $<0.001$ \\
\hline Non-dipper & Yes & 0.293 & $0.180-0.406$ & 0.031 & 0.123 & $0.088-0.158$ & 0.129 \\
\hline Age & $>55$ years & 0.244 & $0.127-0.361$ & 0.058 & & & \\
\hline Sex & Yes & 0.041 & $-0.061-0.155$ & 0.459 & & & \\
\hline Smoking & Yes & 0.313 & $0.191-0.435$ & $<0.001$ & 0.149 & $0.103-0.195$ & $<0.001$ \\
\hline Drinking & Yes & 0.048 & $-0.071-0.167$ & 0.752 & & & \\
\hline Obesity & Yes & 0.196 & $0.113-0.189$ & 0.279 & & & \\
\hline $\mathrm{T} 2 \mathrm{DM}$ & Yes & 0.155 & $0.056-0.254$ & 0.009 & 0.088 & $0.064-0.112$ & 0.021 \\
\hline HLP & Yes & 0.137 & $0.034-0.220$ & 0.026 & 0.063 & $0.052-0.074$ & 0.049 \\
\hline SAHS & Yes & 0.198 & $0.091-0.305$ & 0.055 & & & \\
\hline Hypoxemia & Yes & 0.177 & $0.074-0.280$ & 0.063 & & & \\
\hline CREA & $>92 \mu \mathrm{mol} / 1$ & 0.257 & $0.143-0.371$ & 0.004 & 0.102 & $0.071-0.133$ & 0.012 \\
\hline CRP & $>6.8 \mathrm{mg} / \mathrm{l}$ & 0.134 & $0.031-0.237$ & 0.008 & 0.061 & $0.050-0.072$ & 0.023 \\
\hline Hcy & $>17.9 \mu \mathrm{mol} / 1$ & 0.124 & $0.022-0.228$ & 0.017 & 0.051 & $0.039-0.063$ & 0.046 \\
\hline
\end{tabular}

T2DM, type 2 diabetes mellitus; HLP, hyperlipidemia; SAHS, sleep apnea hypopnea syndrome; CAP, carotid atherosclerotic plaque; CREA, creatinine; CRP, C-reactive protein; Hcy, homocysteine.
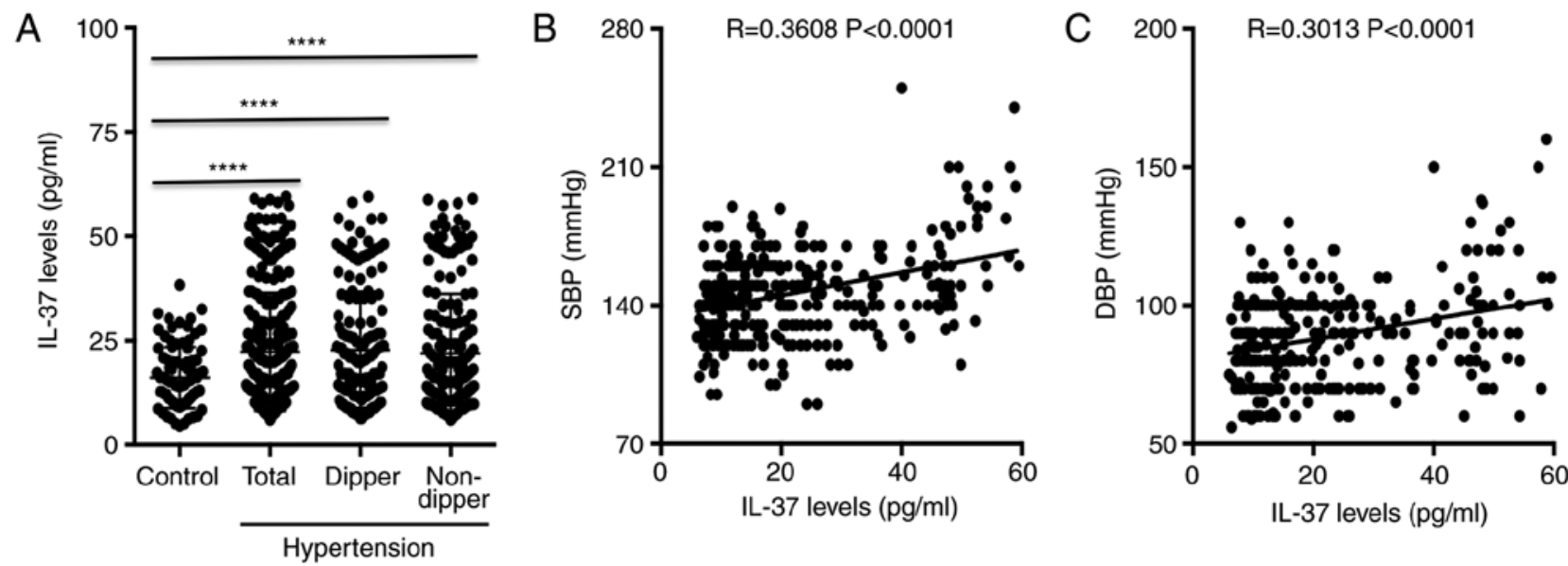

Figure 3. IL-37 levels in dipper and non-dipper hypertension group. (A) IL-37 levels in the control group, the hypertension group, the dipper group and the non-dipper group were measured by ELISA. (B and C) Correlations between the plasma levels of IL-37 and (B) SBP and (C) DBP and in patients with hypertension. ${ }^{* * * * *} \mathrm{P}<0.0001$ vs. the previous group. IL, interleukin; SBP, systolic blood pressure; DBP, diastolic blood pressure.

with ischemic cardiomyopathy, including acute coronary syndrome and acute ST-segment elevation myocardial infarction after percutaneous coronary intervention (26-29). Plasma levels of IL-37 were gradually increased in patients with paroxysmal atrial fibrillation, persistent atrial fibrillation and permanent atrial fibrillation, while the IL-37 levels in all groups were significantly higher than those in the normal group (30). In the present study, the circulating IL-37 concentration was significantly increased in patients with hypertension. These studies may indicate that IL-37 is involved in the development of hypertension. The results also reaffirm the close association between IL-37 and cardiovascular disease.
It is well known that there is a close association between hypertension and immune cells $(20,31)$. Similarly, the differentiation and maturation of immune cells may promote the secretion of various inflammatory factors, which is closely linked to hypertension development $(15,31,32)$. Combined with a previous result by our group indicating that IL-37 may have a regulatory role in the differentiation and maturation of various immune cells and the release of inflammation-associated factors $(13,33)$, it was hypothesized that IL-37 may be involved in the process of hypertension through regulation of inflammatory effects, similar to those that occur in other cardiovascular diseases. The specific role and mechanisms remain to be fully elucidated and still require to be deter- 
A

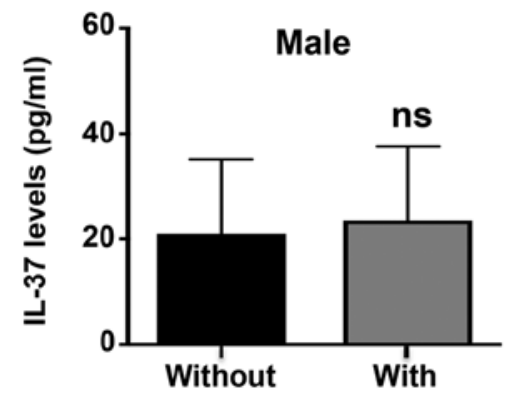

D

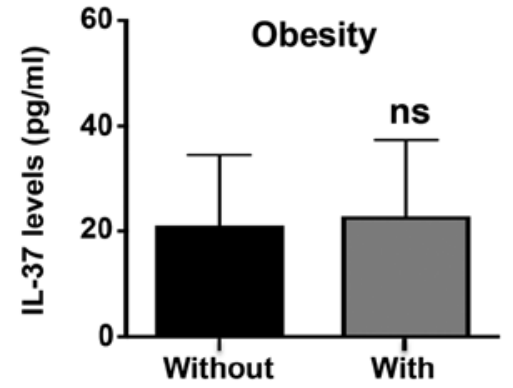

G

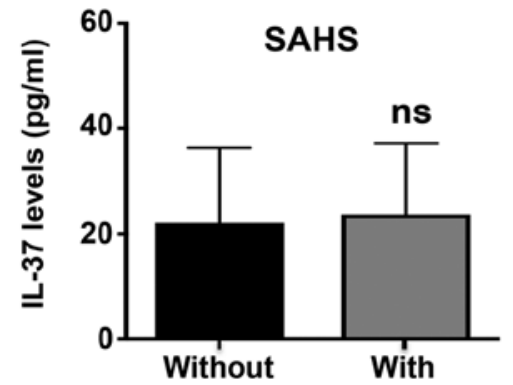

B

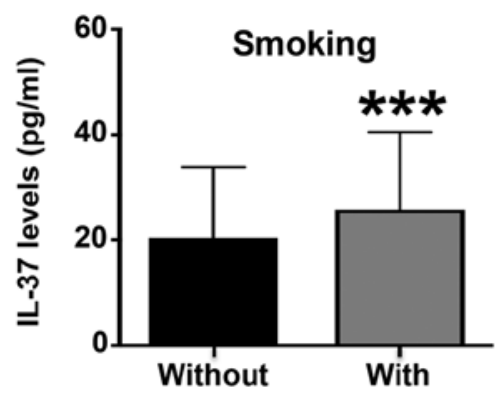

E

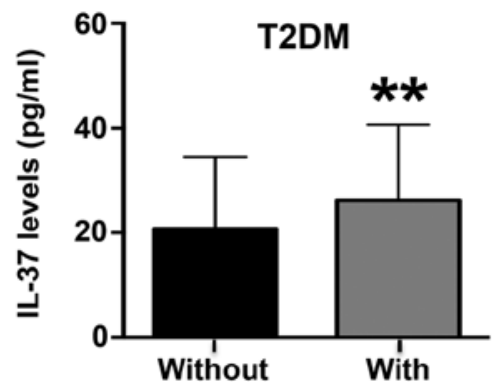

H

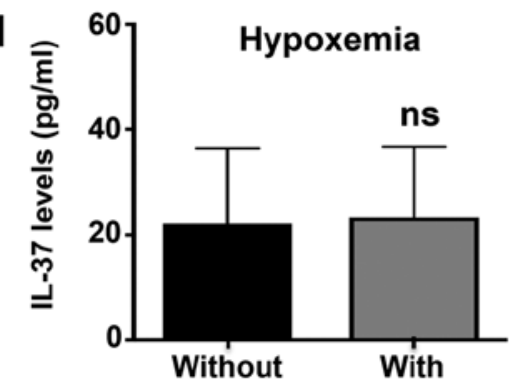

C

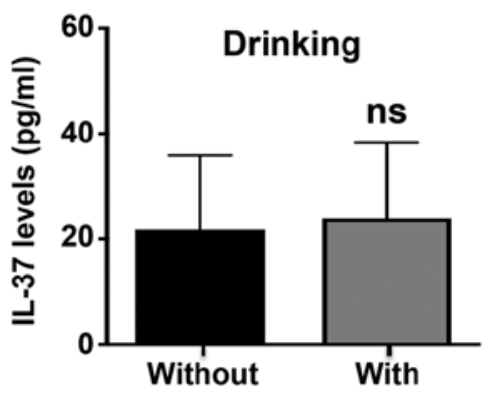

F
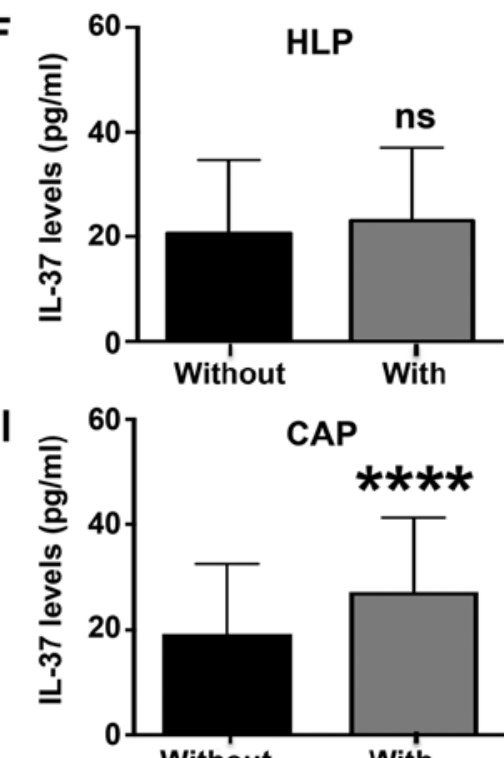

Figure 4. Circulating IL-37 levels in patients with different characteristics. IL-37 levels were determined in patients with hypertension with or without the following characteristics: (A) Male sex, (B) smoking, (C) drinking, (D) obesity, (E) T2DM, (F) HLP, (G) SAHS, (H) hypoxemia and (I) CAP. ** $<0.01$ vs. the without T2DM group; ${ }^{* * *} \mathrm{P}<0.001$ vs. the without smoking group; ${ }^{* * * * *} \mathrm{P}<0.0001$ vs. the without CAP group. ns, no significance; IL, interleukin; T2DM, type 2 diabetes mellitus; HLP, hyperlipidemia; SAHS, sleep apnea hypopnea syndrome; CAP, carotid atherosclerotic plaque.
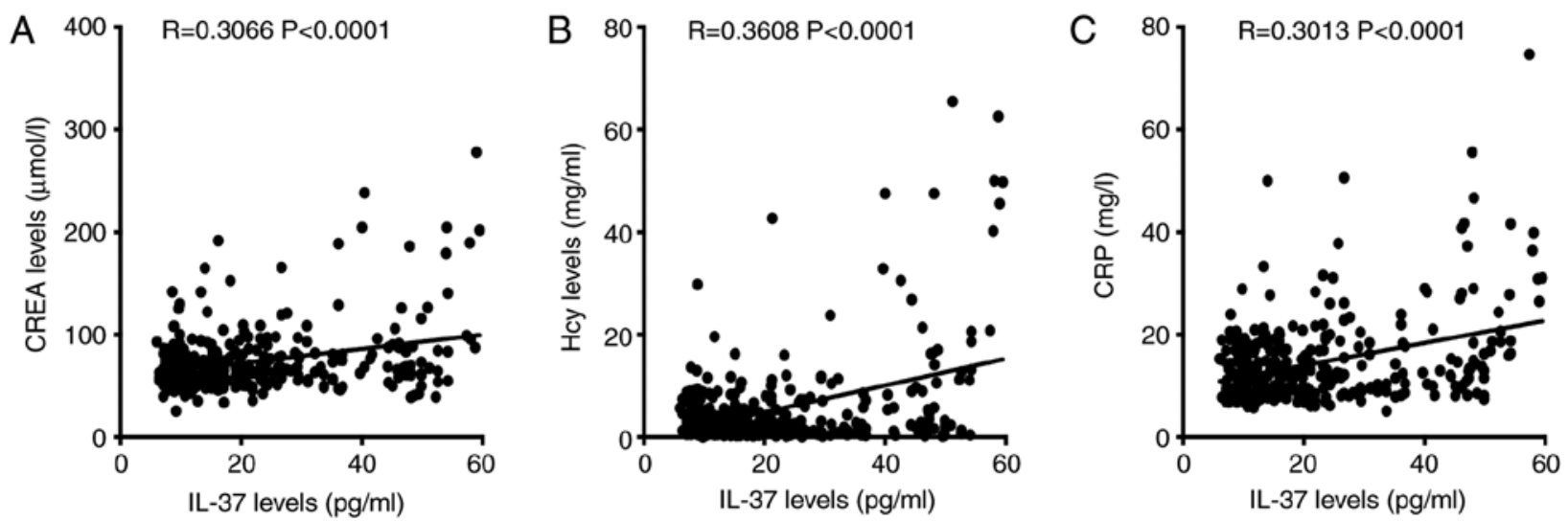

Figure 5. Correlation between IL-37 levels and clinical characteristics. Correlations between IL-37 and (A) CREA, (B) Hcy and (C) CRP were analyzed by Pearson's correlation. IL, interleukin; CREA, creatinine; CRP, C-reactive protein; Hcy, homocysteine.

mined in both animal hypertension studies and clinical experiments.

Hypertension may be divided into dipper hypertension and non-dipper hypertension depending on how much the blood pressure drops at night. Usually, a blood pressure drop of $>10 \%$ at night is referred to as dipper hypertension, which is normal, while a blood pressure drop of $<10 \%$ at night is called non-dipper hypertension, which is abnormal. Several studies have demonstrated that patients with non-dipper hypertension exhibit severe heart, brain and kidney injury, more clinical complications and higher all-cause mortality than patients with dipper hypertension, and this distinction may help predict the prognosis of patients with hypertension $(32,34)$. In the present study, the incidence of CAP was indicated to be significantly lower in patients with dipper hypertension than in patients with non-dipper hypertension. 
Table IV. Influence of various factors including serum IL-37 on the presence of carotid atherosclerotic plaque assessed by univariate analysis and subsequent multivariate linear regression analysis.

\begin{tabular}{|c|c|c|c|c|c|c|c|}
\hline \multirow[b]{2}{*}{ Variable } & \multirow[b]{2}{*}{ Cut-off value } & \multicolumn{3}{|c|}{ Univariate } & \multicolumn{3}{|c|}{ Multivariate } \\
\hline & & Adjusted $\beta$ & $95 \% \mathrm{CI}$ of $\beta$ & P-value & Adjusted $\beta$ & $95 \% \mathrm{CI}$ of $\beta$ & P-value \\
\hline IL-37 & $>30.9 \mathrm{mg} / \mathrm{ml}$ & 0.180 & $0.078-0.280$ & 0.018 & 0.110 & $0.014-0.206$ & 0.024 \\
\hline Hypertension & Yes & 0.183 & $0.081-0.285$ & $<0.001$ & 0.103 & $0.001-0.205$ & 0.047 \\
\hline Non-dipper & Yes & 0.191 & $0.090-0.293$ & $<0.001$ & 0.123 & $0.024-0.223$ & 0.016 \\
\hline Age & $>55$ years & 0.216 & $0.115-0.317$ & $<0.001$ & 0.187 & $0.088-0.286$ & $<0.001$ \\
\hline Sex & Yes & 0.024 & $-0.079-0.126$ & 0.646 & & & \\
\hline Smoking & Yes & 0.282 & $0.183-0.381$ & $<0.001$ & 0.239 & $0.142-0.336$ & $<0.001$ \\
\hline Drinking & Yes & 0.003 & $-0.100-0.107$ & 0.950 & & & \\
\hline Obesity & Yes & 0.083 & $-0.020-0.186$ & 0.115 & & & \\
\hline $\mathrm{T} 2 \mathrm{DM}$ & Yes & 0.147 & $0.044-0.249$ & 0.005 & 0.036 & $-0.066-0.137$ & 0.491 \\
\hline HLP & Yes & 0.124 & $0.022-0.227$ & 0.018 & 0.037 & $-0.060-0.134$ & 0.452 \\
\hline SAHS & Yes & 0.185 & $0.084-0.287$ & $<0.001$ & 0.211 & $-0.045-0.467$ & 0.106 \\
\hline Hypoxemia & Yes & 0.156 & $0.054-0.258$ & 0.003 & -0.084 & $-0.340-0.173$ & 0.522 \\
\hline CREA & $>92 \mu \mathrm{mol} / \mathrm{l}$ & 0.039 & $-0.065-0.142$ & 0.464 & & & \\
\hline CRP & $>6.8 \mathrm{mg} / \mathrm{l}$ & -0.069 & $-0.172-0.034$ & 0.191 & -0.068 & $-0.164-0.027$ & 0.160 \\
\hline Hcy & $>17.9 \mu \mathrm{mol} / \mathrm{l}$ & 0.016 & $-0.088-0.119$ & 0.767 & & & \\
\hline
\end{tabular}

IL, interleukin; T2DM, type 2 diabetes mellitus; HLP, hyperlipidemia; SAHS, sleep apnea hypopnea syndrome; CREA, creatinine; CRP, C-reactive protein; Hcy, homocysteine.

The plasma IL-37 levels in patients with dipper hypertension and non-dipper hypertension were also compared. Of note, there was no significant difference in IL-37 levels between the two groups. These results may suggest that the presence of non-dipper hypertension is associated not with the elevation in circulating IL-37 levels but with other causes. In fact, a previous study reported that the occurrence of non-dipper hypertension was linked to the abnormal increase in blood pressure caused by nocturnal sympathetic nerve excitation (35). These results partially support the hypothesis of the present study.

Numerous clinical characteristics, including smoking, drinking, diabetes, obesity and hyperlipidemia, have been demonstrated to be associated with inflammatory responses and have been indicated to regulate the release of various cytokines, including IL-37 (36,37). These clinical characteristics may promote complex inflammatory responses that may mediate the release of IL-37 by immune cells. To investigate the effects of these clinical characteristics on IL-37 release in patients with hypertension, the patients were divided into two groups based on whether they had these clinical factors and the results suggested that the IL-37 levels were higher in hypertensive patients who smoked, as well as those with T2DM and with CAP. The IL-37 levels were also closely linked to the onset of CAP. These results suggest that elevated IL-37 may have a critical role in inflammatory responses mediated by factors such as smoking and T2DM. As an anti-inflammatory cytokine, IL-37 is increased in a variety of chronic inflammatory diseases. One possible explanation is that IL-37 is increased as a form of feedback to prevent damage mediated by the inflammatory response, just as brain natriuretic peptide is increased in patients with chronic heart failure (38).
There are several limitations to the present study. First, the present study had a single-center design and in the future, studies including samples from multiple centers should be performed. Furthermore, no follow-up was performed to observe the occurrence of complications and determine the impact of IL-37 on prognosis in these patients with hypertension.

In conclusion, the present study indicated that plasma IL-37 levels were increased in patients with hypertension and were positively correlated with blood pressure. IL-37 was also independently associated with the presence of CAP. IL-37 may be a novel therapeutic target for preventing and treating hypertension and prevent organ damage.

\section{Acknowledgements}

The authors would like to thank Dr Jiao Lan from the Molecular Biology Laboratory of Guangxi Zhuang Autonomous Region People's Hospital for their technical support on dendritic cells stimulation and differentiation.

\section{Funding}

This work was supported by the National Natural Science Foundation of China (grant nos. 81560085 and 81770472 to QJ and grant no. 81760051 to YL).

\section{Availability of data and materials}

The datasets used and/or analyzed during the current study are available from the corresponding author on reasonable request. 


\section{Authors' contributions}

YL, JW and QJ conceived and designed the study; JY and YW collected the samples; JY, YW, ZW, YL, LL, QZ, MW, YX, DY, JZ, JW and QJ (all authors) performed the experiments; ZW and QZ analyzed the data; MW and YX were involved in drafting the manuscript or revising it critically for important intellectual content; DY and JZ reviewed and edited the manuscript; QJ and JW gave final approval of the version to be published. All authors read and approved the final manuscript.

\section{Ethics approval and consent to participate}

The study conformed to the guidelines approved by the Ethics Committee of the People's Hospital of Guangxi Zhuang Autonomous Region (Nanning, China; approval no. 2015-16 of the National Natural Science Foundation of China).

\section{Patient consent for publication}

Not applicable.

\section{Competing interests}

The authors declare that they have no competing interests.

\section{References}

1. Laurent S and Boutouyrie P: The structural factor of hypertension: Large and small artery alterations. Circ Res 116 1007-10021, 2015.

2. Guzik TJ and Touyz RM: Oxidative stress, inflammation, and vascular aging in hypertension. Hypertension 70: 660-667, 2017.

3. Shirakabe A, Ikeda Y, Sciarretta S, Zablocki DK and Sadoshima J: Aging and autophagy in the heart. Circ Res 118: 1563-1576, 2016.

4. Boraschi D, Lucchesi D, Hainzl S, Leitner M, Maier E, Mangelberger D, Oostingh GJ, Pfaller T, Pixner C, Posselt G, et al: IL-37: A new anti-inflammatory cytokine of the IL-1 family. Eur Cytokine Netw 22: 127-147, 2011.

5. Busfield SJ, Comrack CA, Yu G, Chickering TW, Smutko JS, Zhou H, Leiby KR, Holmgren LM, Gearing DP and Pan Y: Identification and gene organization of three novel members of the IL-1 family on human chromosome 2. Genomics 66: 213-216, 2000.

6. Dinarello CA, Nold-Petry C, Nold M, Fujita M, Li S, Kim S and Bufler P: Suppression of innate inflammation and immunity by interleukin-37. Eur J Immunol 46: 1067-1081, 2016.

7. Nold-Petry CA, Lo CY, Rudloff I, Elgass KD, Li S, Gantier MP, Lotz-Havla AS, Gersting SW, Cho SX, Lao JC, et al: IL-37 requires the receptors IL-18R $\alpha$ and IL-1R8 (SIGIRR) to carry out its multifaceted anti-inflammatory program upon innate signal transduction. Nat Immunol 16: 354-365, 2015.

8. Nold MF, Nold-Petry CA, Zepp JA, Palmer BE, Bufler P and Dinarello CA: IL-37 is a fundamental inhibitor of innate immunity. Nat Immunol 11: 1014-1022, 2010.

9. Zhuang X, Wu B, Li J, Shi H, Jin B and Luo X: The emerging role of interleukin-37 in cardiovascular diseases. Immun Inflamm Dis 5: 373-379, 2017.

10. Jia H, Liu J and Han B: Reviews of interleukin-37: Functions, receptors, and roles in diseases. Biomed Res Int 2018: 3058640, 2018.

11. Ji QW, Meng K, Yu KW, Huang S, Huang Y, Min XH, Zhong YC, Wu BW, Liu YZ, Nie SP, et al: Exogenous interleukin 37 ameliorates atherosclerosis via inducing the Treg response in ApoE-deficient mice. Sci Rep 7: 3310, 2017.

12. Liu J, Lin J, He S, Wu C, Wang B, Liu J, Duan Y, Liu T, Shan S, Yang $\mathrm{K}$, et al: Transgenic overexpression of IL-37 protects against atherosclerosis and strengthens plaque stability. Cell Physiol Biochem 45: 1034-1050, 2018.
13. Liu T, Liu J, Lin Y, Que B, Chang C, Zhang J, Liang Z, Gao X, Liu S, Liu L, et al: IL-37 inhibits the maturation of dendritic cells through the IL-1R8-TLR4-NF- $\mathrm{B}$ pathway. Biochim Biophys Acta Mol Cell Biol Lipids 1864: 1338-1349, 2019.

14. Hoeke G, Khedoe PP, van Diepen JA, Pike-Overzet K, van de Ven B, Vazirpanah N, Mol I, Hiemstra PS, Staal FJ, Stienstra R, et al: The effects of selective hematopoietic expression of human IL-37 on systemic inflammation and atherosclerosis in LDLr-deficient mice. Int J Mol Sci 18: 1672, 2017.

15. Zhu R, Sun H, Yu K, Zhong Y, Shi H, Wei Y, Su X, Xu W, Luo Q, Zhang F, et al: Interleukin-37 and dendritic cells treated with interleukin-37 plus Troponin I ameliorate cardiac remodeling after myocardial infarction. J Am Heart Assoc 5: e004406, 2016.

16. Wu B, Meng K, Ji Q, Cheng M, Yu K, Zhao X, Tony H, Liu Y, Zhou Y, Chang C, et al: Interleukin-37 ameliorates myocardial Ischaemia/reperfusion injury in mice. Clin Exp Immunol 176: 438-451, 2014.

17. Ji Q, Cheng G, Ma N, Huang Y, Lin Y, Zhou Q, Que B, Dong J, Zhou Y and Nie S: Circulating Th1, Th2, and Th17 levels in hypertensive patients. Dis Markers 2017: 7146290, 2017.

18. Postel-Vinay N, Steichen O, Pébelier E, Persu A, Berra E, Bobrie G, Savard S, Nogueria J and Azizi M: Home blood pressure monitoring and e-Health: Investigation of patients experience with the Hy-Result system. Blood Press Monit 25: $155-161,2020$.

19. Rimoldi SF, Scherrer U and Messerli FH: Secondary arterial hypertension: When, who, and how to screen? Eur Heart J 35: 1245-1254, 2014.

20. Ye J, Que B, Huang Y, Lin Y, Chen J, Liu L, Shi Y, Wang Y, Wang M, Zeng T, et al: Interleukin-12p35 knockout promotes macrophage differentiation, aggravates vascular dysfunction, and elevates blood pressure in angiotensin II-infused mice. Cardiovase Res 115: 1102-1113, 2019.

21. Livak KJ and Schmittgen TD: Analysis of relative gene expression data using real-time quantitative PCR and the 2(-Delta Delta C(T)) method. Methods 25: 402-408, 2001.

22. Yu K, Min X, Lin Y, Huang Y, Huang S, Liu L, Peng Y, Meng K, Li D, Ji Q and Zeng Q: Increased IL-37 concentrations in patients with arterial calcification. Clin Chim Acta 461: 19-24, 2016.

23. Chai M, Zhang HT, Zhou YJ, Ji QW, Yang Q, Liu YY, Zhao YX, Shi DM, Liu W, Yang LX, et al: Elevated IL-37 levels in the plasma of patients with severe coronary artery calcification. J Geriatr Cardiol 14: 285-291, 2017.

24. Kapelouzou A, Kontogiannis C, Tsilimigras DI, Georgiopoulos G, Kaklamanis L, Tsourelis L and Cokkinos DV: Differential expression patterns of toll like receptors and interleukin-37 between calcific aortic and mitral valve cusps in humans. Cytokine 116: 150-160, 2019.

25. Shou X, Lin J, Xie C, Wang Y and Sun C: Plasma IL-37 elevated in patients with chronic heart failure and predicted major adverse cardiac events: A 1-year follow-up study. Dis Markers 2017: 9134079, 2017.

26. Ji Q, Zeng Q, Huang Y, Shi Y, Lin Y, Lu Z, Meng K, Wu B, Yu K, Chai M, et al: Elevated plasma IL-37, IL-18, and IL-18BP concentrations in patients with acute coronary syndrome. Mediators Inflamm 2014: 165742, 2014.

27. Wang X, Cai X, Chen L, Xu D and Li J: The evaluation of plasma and leukocytic IL-37 expression in early inflammation in patients with acute ST-Segment elevation myocardial infarction after PCI. Mediators Inflamm 2015: 626934, 2015.

28. Yang T, Fang F, Chen Y, Ma J, Xiao Z, Zou S, Zheng N, Yan D, Liao S, Chen S, et al. Elevated plasma interleukin-37 playing an important role in acute coronary syndrome through suppression of ROCK activation. Oncotarget 8: 9686-9695, 2017.

29. Mao X, Zhu R, Zhang F, Zhong Y, Yu K, Wei Y, Sun H, Xu W, Luo Q, Wang Y, et al: IL-37 plays a beneficial role in patients with acute coronary syndrome. Mediators Inflamm 2019: 9515346, 2019.

30. Li W, Li S, Li X, Jiang S and Han B: Interleukin-37 elevation in patients with atrial fibrillation. Clin Cardiol 40: 66-72, 2017.

31. Kirabo A, Fontana V, de Faria AP, Loperena R, Galindo CL, Wu J, Bikineyeva AT, Dikalov S, Xiao L, Chen W, et al: DC isoketal-modified proteins activate $\mathrm{T}$ cells and promote hypertension. J Clin Invest 124: 4642-4656, 2014.

32. Aksit E, Gursul E, Aydin F, Samsa M and Ozcelik F: Non-dipper hypertension is associated with slow coronary flow among hypertensives with normal coronary angiogram. Cardiovasc J Afr 28: 14-18, 2017. 
33. Ji Q, Meng K, Yu K, Huang S, Huang Y, Min X, Zhong Y, Wu B, Liu Y, Nie S, et al: Exogenous interleukin 37 ameliorates atherosclerosis via inducing the Treg response in ApoE-deficient mice. Sci Rep 7: 3310, 2017

34. Chen Y, Liu JH, Zhen Z, Zuo Y, Lin Q, Liu M, Zhao C, Wu M, Cao G, Wang R, et al: Assessment of left ventricular function and peripheral vascular arterial stiffness in patients with dipper and non-dipper hypertension. J Investig Med 66: 319-324, 2018

35. Ragot S, Herpin D, Siché JP, Ingrand $P$ and Mallion JM: Autonomic nervous system activity in dipper and non-dipper essential hypertensive patients. What about sex differences? J Hypertens 17: 1805-1811, 1999.

36. Di Stefano A, Caramori G, Barczyk A, Vicari C, Brun P,Zanini A, Cappello F, Garofano E, Padovani A, Contoli M, et al: Innate immunity but not NLRP3 inflammasome activation correlates with severity of stable COPD. Thorax 69: 516-524, 2014.
37. Borzouei S, Sheikh V, Ghasemi M, Zamani A, Telikani Z, Zareighane Z, Salehi I, Mozayanimonfared A, Amirzargar MA and Alahgholi-Hajibehzad M: Anti-inflammatory effect of combined sitagliptin and vitamin D3 on cytokines profile in patients with type 2 diabetes mellitus. J Interferon Cytokine Res 39: 293-301, 2019.

38. Ye J, Wang Z, Ye D, Wang Y, Wang M, Ji Q, Huang Y, Liu L, Shi Y, Shi L, et al: Increased interleukin-11 levels are correlated with cardiac events in patients with chronic heart failure. Mediators Inflamm 2019: 1575410, 2019. 